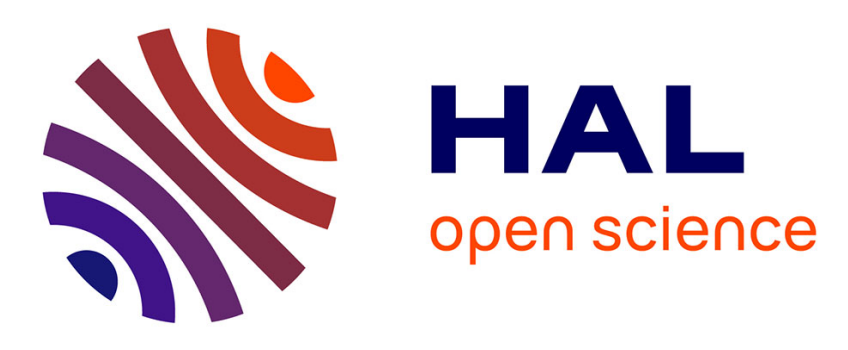

\title{
A simple and fast technique to analyze accurately reconfigurable reflectarrays
}

Clement Yann, Renaud Loison, Raphaël Gillard, Michèle Labeyrie, Jean-Paul Martinaud

\section{- To cite this version:}

Clement Yann, Renaud Loison, Raphaël Gillard, Michèle Labeyrie, Jean-Paul Martinaud. A simple and fast technique to analyze accurately reconfigurable reflectarrays. European Conference on Antennas and Propagation, Mar 2012, Czech Republic. pp. 930-933. hal-00768650

\section{HAL Id: hal-00768650 https://hal.science/hal-00768650}

Submitted on 22 Dec 2012

HAL is a multi-disciplinary open access archive for the deposit and dissemination of scientific research documents, whether they are published or not. The documents may come from teaching and research institutions in France or abroad, or from public or private research centers.
L'archive ouverte pluridisciplinaire HAL, est destinée au dépôt et à la diffusion de documents scientifiques de niveau recherche, publiés ou non, émanant des établissements d'enseignement et de recherche français ou étrangers, des laboratoires publics ou privés. 


\section{A Simple and Fast Technique to Analyze Accurately Reconfigurable Reflectarrays}

\author{
C. Yann, R. Loison, R. Gillard \\ European University of Brittany, France, INSA, IETR, \\ UMR 6164, F-35708 Rennes \\ Clement.Yann@insa-rennes.fr \\ Renaud.Loison@insa-rennes.fr \\ Raphael.Gillard@insa-rennes.fr
}

\begin{abstract}
A simple and fast technique is proposed and validated to analyze reconfigurable reflectarrays. This technique takes into account mutual coupling effects between active cells within the array. Its novelty relies on the ease of use, the accuracy and the promptness for analyzing reconfigurable reflectarrays.
\end{abstract}

Reconfigurable reflectarray; mutual coupling; array factor; analysis method

\section{INTRODUCTION}

Reconfigurable reflectarray antennas (RRA) [1]-[2] are a promising solution for space communications and radar applications but also for RFID [3] and optoelectronic applications. RRA is an array of active cells, which is illuminated by a primary feed source. The active cells reflect the incident wave with prescribed phase shifts to form a desired radiation pattern. All the cells have the same geometry and they differ only by active element states. An active cell is able to adaptively change the phase of the radiated wave for instance by electronically switching active elements such as PIN diodes [4] or MEMS [2].

Reflectarray analysis is a major issue due to the complexity of cell geometry and the size of the whole array.

For passive reflectarrays, two commonly used methods are the Floquet approach [5], and the 'surrounded-cell' approach [6]. The first one assumes that the unitary cell is extracted from an infinite periodic array of identical cells. It approximates mutual coupling because the cells within a realistic reflectarray are different. The 'surrounded-element' approach simulates each radiating cell with its actual neighboring cells. The mutual coupling effects are, in this case, realistically accounted for.

For RRA, a method, based on the 'surrounded-element' approach, has been proposed in [7]. The main advantage of this method is that it takes into account mutual coupling effects. It requires only one lightweight electromagnetic simulation for the whole RRA and its efficiency has been illustrated on a RRA example. In this paper, we propose to improve this method by drastically reducing the required database size and the computation time while preserving the accuracy.

This work is supported by Thales Systèmes Aéroportés, Elancourt, France.

\author{
M. Labeyrie, J-P Martinaud \\ THALES Systèmes Aéroportés \\ Elancourt, France \\ michele.labeyrie@fr.thalesgroup.com \\ jean-paul.martinaud@fr.thalesgroup.com
}

\section{PRINCIPLE}

\section{A. Analysis method}

As detailed in [7], each cell in the array is studied one after the other to compute the reflectarray radiation pattern. To do so, a sub-array made up of the considered cell and its closest neighbors is considered (Fig. 1).

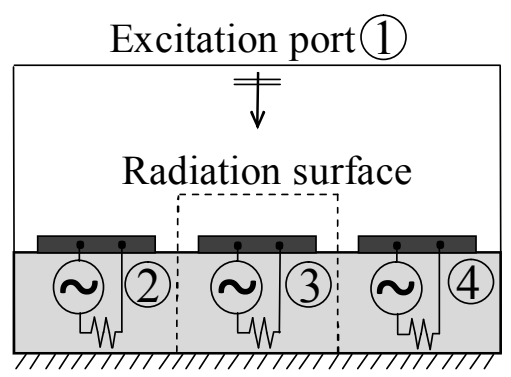

Fig. 1. Electromagnetic simulation of the sub-array.

For the sake of simplicity, only the two closest neighbors are considered here and each cell includes only one active element. The whole sub-array is illuminated by the incident wave and the far-field radiated from the central cell is determined. The specific state of active elements within the considered cell and its neighbors is taken into account through circuit simulations. Using the superposition principle, the radiation pattern of the overall array is then constructed by summing all the unit-cell radiation patterns. Even if the method is accurate, the storage of a large number of radiation patterns is required for each incidence and polarization. This large quantity of data complicates the implementation of the method.

\section{B. Proposed improvement}

In the proposed improvement, only the phase response of each active cell considering the actual environment is computed which is time-saving and provides ease of use and accuracy.

Firstly, the phase response of each active cell surrounded by its actual neighbors is computed. Secondly, the array-factor of the overall RRA is computed, from the actual phase of each active cell, with a phase-only control. In the meantime, the 
unit-cell radiation pattern is determined by simulating a simplified model of the unit-cell. Finally, the radiation pattern of the overall RRA is given by the product of the unit-cell radiation pattern and the array factor.

\section{APPLICATION}

Two examples are considered to demonstrate the performance and the applicability of the proposed technique for analyzing RRA.

\section{A. Description of the unit-cell}

THALES Systèmes Aéroportés has developed a RRA and a detailed description of the cell can be found in [8]. The unitcell consists of a printed circuit with 4 diodes inside an open rectangular metallic cavity as shown on Fig. 2.

With the two pairs of diodes located on the printed circuit, the active cell exhibits 4 phase values (Table I). Diodes have a forward resistance of $2.5 \mathrm{Ohms}\left(\mathrm{Z}_{\mathrm{On}}\right)$ while their reverse blocking capacitance $\left(\mathrm{Z}_{\mathrm{Off}}\right)$ is $50 \mathrm{fF}$.

The cells are geometrically identical but the diodes are switched in different states to control the far-field characteristics. The electromagnetic HFSS ${ }^{\circledR}$ commercial software, which is based on the Finite Element Method, is used and the unit-cell design used for the simulation is represented in Fig. 3.

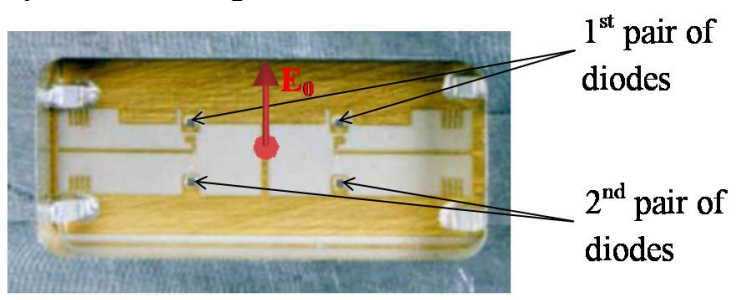

Fig. 2. Reflectarray phase shifting cell

TABLE I

PHASES OF THE CELL UNDER NORMAL INCIDENCE AT 8.7GHZ WITH THE FLOQUET APPROACH

\begin{tabular}{|l|l|}
\hline State & Phase \\
\hline On-On & $31.0^{\circ}$ \\
\hline Off-Off & $-49.0^{\circ}$ \\
\hline On-Off & $-123.6^{\circ}$ \\
\hline Off-On & $-175.8^{\circ}$ \\
\hline
\end{tabular}

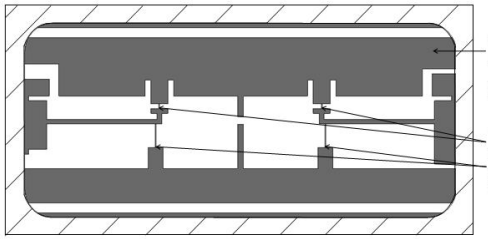

Printed circuit Pairs of diodes

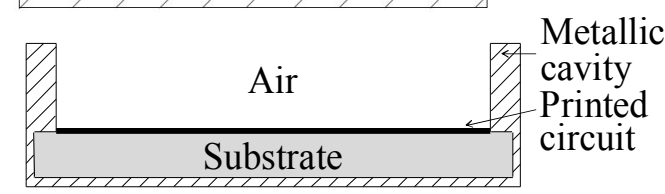

Fig. 3. Unit-cell design for the simulation

\section{B. Implementation of the proposed technique}

As described in the principle of the proposed technique, the phase response of each active cell is determined considering the actual neighbors. In the two examples, 24 neighbors around each active cell are considered, that is to say $5 \times 5$ sub-arrays. In [7], it has been validated that this size of sub-arrays is sufficient to consider mutual coupling effects in RRA made up of this type of cells. However, the technique can be applied to larger sub-arrays for other RRA.

In order to obtain the radiation pattern of the overall RRA, the array factor is computed from the actual phase responses of each active cell. The phase response corresponds actually to the phase of the co-polarization component of the electric field in the specular direction.

Next, the array factor is multiplied by the unit-cell radiation pattern. The radiation pattern of an open rectangular waveguide is used to approximate the cell's pattern. It corresponds to the unit-cell design as depicted in Fig. 3 with the printed circuit replaced by a perfect electric conductor.

\section{Analysis of a $10 \times 10$ difference array}

A small-sized array of 10x10 cells is studied so that it can be simulated entirely with HFSS $^{\circledR}$ for validation.

The array is illuminated by a normal-incident plane wave and the desired radiation pattern has a null in the $\theta=0^{\circ}$ direction and a main beam on each side of this null of radiation. To get these characteristics of the radiation pattern, the diodes are switched in a specific state. A quasi differencepattern is obtained by using the "On-On" state for the diodes on the upper half of the array and the "Off-On" state for those on the lower half. Then, there is $180^{\circ}$ out of phase from each part resulting in a null of amplitude at $\theta=0^{\circ}$.

The RRA is analyzed with different approaches (Fig. 5). Firstly, a HFSS ${ }^{\circledR}$ full-wave electromagnetic simulation of the overall RRA, which is the reference simulation, is carried out. Secondly, the radiation pattern of the overall RRA is computed using the proposed technique. Thirdly, the radiation pattern of the overall RRA is computed using the Floquet phase of each active cell. This pattern is obtained by multiplying the unit-cell radiation pattern and an array factor computed with the Floquet phases.

The results produced by the proposed technique are in good agreement with those obtained with the reference simulation. Moreover, the proposed technique gives better results than the Floquet approach. 


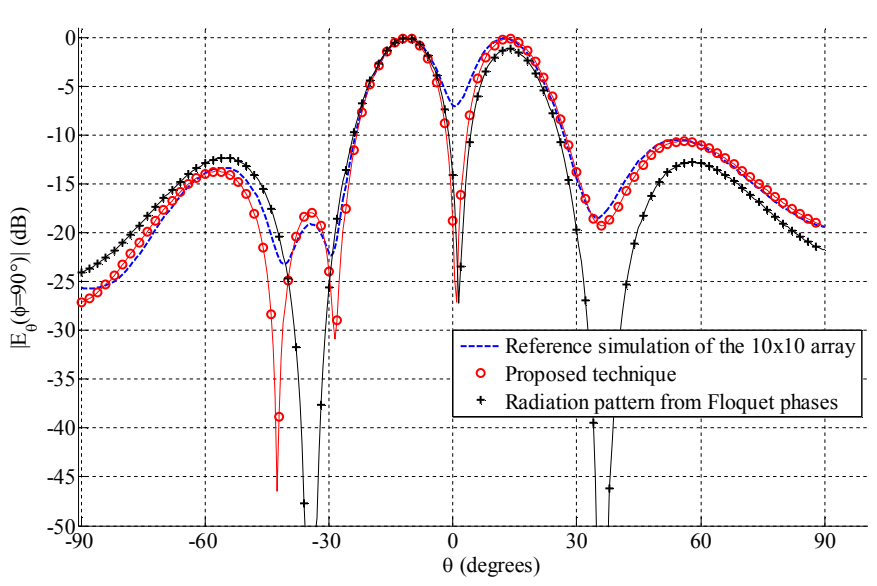

Fig. 5. Radiation patterns of the $10 \times 10$ difference array.

The statistical results set out in Table II compare the different approaches to the reference simulation. The $\mathrm{RL}_{\text {error }}$ parameter estimates the amplitude difference of the right main lobe between the studied technique and the reference $\left(\theta=14^{\circ}\right.$ direction). This error is $1.07 \mathrm{~dB}$ for Floquet and $0.004 \mathrm{~dB}$ for the proposed technique. The MAE parameter, which stands for "Mean Absolute Error", is an average of the absolute differences between the studied technique and the reference over the $\left[-90^{\circ} ; 90^{\circ}\right]$ range. An improvement is observed with the proposed technique. However, in this case, the Floquet approach is still acceptable given that the array can be seen as the juxtaposition of two periodic arrays.

TABLE II

STATISTICAL RESULTS OF THE 10X10 DIFFERENCE ARRAY

\begin{tabular}{|l|l|l|}
\cline { 2 - 3 } \multicolumn{1}{c|}{} & Floquet & $\begin{array}{l}\text { Proposed } \\
\text { technique }\end{array}$ \\
\hline $\mathrm{RL}_{\text {error }}(\mathrm{dB})$ & 1.07 & 0.004 \\
\hline $\mathrm{MAE}(\mathrm{dB})$ & 4.41 & 1.31 \\
\hline
\end{tabular}

The purpose of the following example is to show that the proposed method is more robust than the Floquet approach when irregularities appear in the array regarding the diode states.

\section{Analysis of a 10x10 RRA with a main beam in the $\left(\varphi_{\max }=\right.$ $\left.45^{\circ} ; \theta_{\max }=30^{\circ}\right)$ direction}

A second RRA composed of 100 cells is considered. The array is illuminated by a normal-incident plane wave and the aim is to steer the direction of the main beam in the $\left(\varphi_{\max }=\right.$ $45^{\circ} ; \theta_{\max }=30^{\circ}$ ) direction at $8.7 \mathrm{GHz}$. The state of all diodes is determined according to the relevant phase law using Floquet phases (Table I) and the configuration of diodes is depicted in Fig. 6.
$\mathbf{Y}_{4}$

\begin{tabular}{|l|l|l|l|l|l|l|l|l|l|}
\hline On On & Off Off & Off Off & On Off & On Of & Off On & Off On & On On & On On & On On \\
\hline Off Off & On Off & On Off & Off On & Off On & On On & On On & Off Off & Off Off & On Off \\
\hline Off On & Off On & Off On & On On & On On & Off Off & Off Off & On Off & On Off & Off On \\
\hline Off On & On On & On On & Off Off & Off Off & On Off & On Off & Off On & Off On & On On \\
\hline On On & Off Off & Off Off & On Off & Off On & Off On & Off On & On On & On On & Off Off \\
\hline Off Off & On Off & Off On & Off On & Off On & On On & On On & Off Off & Off Off & On Off \\
\hline Off On & Off On & Off On & On On & On On & Off Off & Off Off & On Off & Off On & Off On \\
\hline Off On & On On & On On & Off Off & Off Off & On Off & Off On & Off On & Off On & On On \\
\hline On On & Off Off & Off Off & On Off & Off On & Off On & Off On & On On & On On & Off Off \\
\hline Off Off & On Off & Off On & Off On & Off On & On On & On On & Off Off & Off Off & On Off \\
\hline
\end{tabular}

Fig. 6. $10 \times 10$ RRA with a main beam in the $\left(\varphi_{\max }=45^{\circ} ; \theta_{\max }=30^{\circ}\right)$ direction

The RRA is analyzed with the same three approaches as in the previous example. The radiation patterns are reported in Fig. 7.

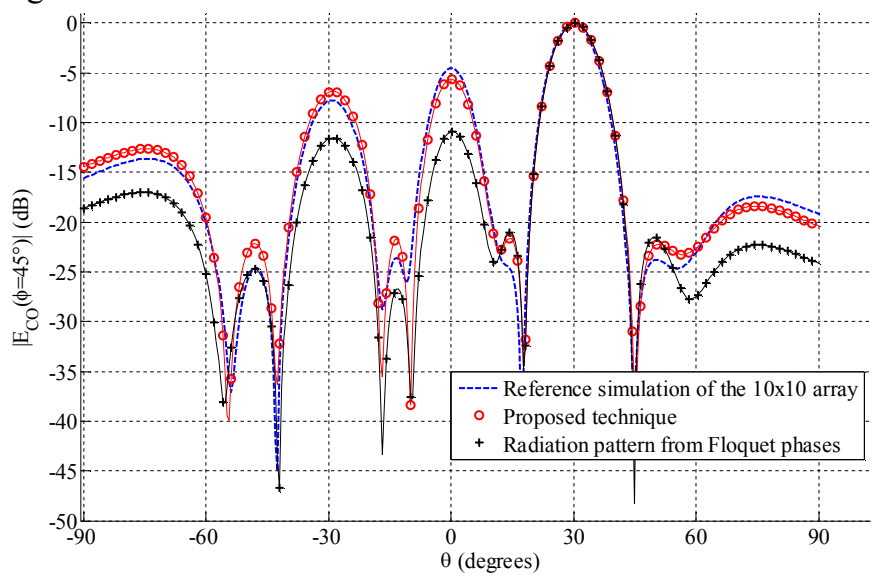

Fig. 7. Radiation patterns of the 10x10 RRA.

The statistical results set out in Table III reveal significant differences between the Floquet approach and the reference. On the contrary, the results obtained with the proposed technique are in good agreement with the reference. The $\mathrm{FQL}_{\text {error }}$ parameter estimates the amplitude difference of the first quantification lobe between the studied technique and the reference. This error is $6.43 \mathrm{~dB}$ for Floquet and $1.13 \mathrm{~dB}$ for the proposed technique. On the second quantification lobe (SQL), the error is $3.82 \mathrm{~dB}$ for Floquet and less than $1 \mathrm{~dB}$ for the proposed technique. The MAE is equal to $3.75 \mathrm{~dB}$ with the Floquet approach and to $1.39 \mathrm{~dB}$ with the proposed technique. As a conclusion, the results obtained with the proposed technique agree satisfactorily with the reference results whereas the Floquet approach does not predict the quantification lobes levels with accuracy.

TABLE III

STATISTICAL RESULTS OF THE 10X10 RRA

\begin{tabular}{|l|l|l|}
\cline { 2 - 3 } \multicolumn{1}{c|}{} & Floquet & $\begin{array}{l}\text { Proposed } \\
\text { technique }\end{array}$ \\
\hline $\mathrm{FQL}_{\text {error }}(\mathrm{dB})$ & 6.43 & 1.13 \\
\hline $\mathrm{SQL}_{\text {error }}(\mathrm{dB})$ & 3.82 & 0.85 \\
\hline $\mathrm{MAE}(\mathrm{dB})$ & 3.75 & 1.39 \\
\hline
\end{tabular}




\section{E. Computation time}

All the simulations are carried out on an Intel ${ }^{\circledR}$ Xeon ${ }^{\circledR}$ E5506 2.13GHz Quad Core with 48 GB RAM. The reference simulation requires 587 minutes for each configuration of active elements. In comparison, for the first configuration, the proposed technique requires 201 minutes for the electromagnetic simulation and the post-processing requires 50 seconds for the $3 \mathrm{D}$ radiation pattern. For other configurations, only the 50 seconds of the post-processing are required given that the same electromagnetic simulation can be used.

\section{F. Database requirements}

As previously mentioned, the complete analysis method presented in [7] requires the storage of a large number of radiation patterns. This large quantity of data complicates the implementation of the method. The main advantage of the proposed technique is that the size of the database is drastically reduced.

The database requirements for the complete analysis method and the proposed technique are given in Table IV. The complex E-fields correspond to the required radiation patterns. For the proposed technique, only the radiation pattern of an open rectangular waveguide is required, that is to say 361 complex E-field values over the $\left[-90^{\circ}, 90^{\circ}\right]$ range. The complex voltages and currents are a specification of the HFSS implementation and are used to determine the S-parameters.

For the complete analysis method, the required database size is 911725 complex values for only one plane and one electric field component. In comparison, the proposed technique required only 2725 values for the $3 \mathrm{D}$ radiation pattern. As a consequence, the database size is significantly reduced thanks to the proposed technique.

\section{CONCLUSION}

A simple and fast technique for analyzing RRA has been described and validated. The results show clearly that the proposed technique is more accurate than the Floquet approach. Besides, this technique leads to a drastic reduction of the computational costs given that it requires only one electromagnetic simulation and light post-processing computations.
TABLE IV

SIZE OF THE DATABASE

\begin{tabular}{|c|c|c|}
\hline & \multicolumn{2}{|c|}{ SIZE OF THE DATABASE } \\
\hline & $\begin{array}{l}\text { Complete analysis } \\
\text { method }\end{array}$ & $\begin{array}{l}\text { Proposed } \\
\text { technique }\end{array}$ \\
\hline $\begin{array}{l}\text { Complex E- } \\
\text { Fields }\end{array}$ & $\begin{array}{c}\mathrm{n} \theta *(\mathrm{nDiodes}+\mathrm{nInc} \\
) * \mathrm{nRadSurf}= \\
911525\end{array}$ & $\mathrm{n} \theta=361$ \\
\hline Phase responses & & $\begin{array}{c}\text { (nDiodes+nInc } \\
\text { )*nRadSurf } \\
=2525\end{array}$ \\
\hline $\begin{array}{l}\text { Complex } \\
\text { voltages }\end{array}$ & \multicolumn{2}{|c|}{ nDiodes $*$ nInc $=100$} \\
\hline $\begin{array}{l}\text { Complex } \\
\text { currents }\end{array}$ & \multicolumn{2}{|c|}{ nDiodes $*$ nInc $=100$} \\
\hline Total & $\begin{array}{l}911725 \\
\text { For } 1 \text { plane / } 1 \\
\text { E-Field component }\end{array}$ & $\begin{array}{c}2725 \\
\text { For the } 3 \mathrm{D} \\
\text { radiation } \\
\text { pattern }\end{array}$ \\
\hline
\end{tabular}

$\mathrm{n} \theta$ : number of $\theta$ values over the $\left[-90^{\circ}, 90^{\circ}\right]$ range.

nDiodes: number of diodes in the array.

nInc: number of incidences and polarizations.

nRadSurf: number of radiation surfaces ( 25 for a $5 \times 5$ sub-array).

\section{REFERENCES}

[1] J. Huang, and J. Encinar, Reflectarray Antennas. Wiley-IEEE Press, 2007.

[2] J. Perruisseau-Carrier, "Recent developments in reconfigurable reflectarrays," in ICECom, 2010 Conference Proceedings, Dubrovnik, Croatia, Sept. 2010, pp. 1-4.

[3] Hsi-Tseng Chou, Tso-Ming Hung, Nan-Nan Wang, Hsi-Hsir Chou, Chia Tung, and P. Nepa, "Design of a Near-Field Focused Reflectarray Antenna for $2.4 \mathrm{GHz}$ RFID Reader Applications", IEEE Trans. on Antennas and Propagation, vol. 59, no. 3, pp. 1013-1018, Mar. 2011.

[4] L. Boccia, F. Venneri, G. Amendola, G. Di Massa, "Application of varactor diodes for reflectarray phase control," in IEEE Antennas and Propagation Society International Symposium, San Antonio, Texas, 2002, vol. 3, p. 132.

[5] J. Montgomery, "Scattering by an infinite periodic array of microstrip elements," IEEE Trans. on Antennas and Propagation, vol. 26, no. 6, pp. 850-854, Nov. 1978.

[6] M.-A. Milon, D. Cadoret, R. Gillard, and H. Legay, "Surroundedelement approach for the simulation of reflectarray radiating cells," IET Microwaves, Antennas \& Propagation, vol. 1, no. 2, pp. 289-293, Apr. 2007.

[7] C. Yann, R. Loison, R. Gillard, M. Labeyrie, and J.-P. Martinaud, "Global technique analysis for reconfigurable reflectarray antennas," in European Conference on Antennas and Propagation, Rome, Italy, 2011.

[8] X. Delestre, T. Dousset, M. Labeyrie, and C. Renard, "New Challenges for Active ReflectArrays," in International Radar Conference, Bordeaux, France, 2009. 